\title{
MicroMegas-based detectors for time-of-flight measurements of neutron-induced reactions
}

\author{
F. Gunsing ${ }^{1, *}$, F. Belloni ${ }^{1}$, E. Berthoumieux ${ }^{1}$, M. Diakaki ${ }^{2}$, E. Dupont ${ }^{1}$, E. Ferrer-Ribas ${ }^{1}$, and Th. Papaevangelou ${ }^{1}$ \\ ${ }^{1}$ CEA - Irfu, University Paris-Saclay, F-91191 Gif-sur-Yvette, France \\ ${ }^{2}$ CEA Cadarache, DEN/DER/SPRC/LEPh, F-13108 St-Paul-Lez-Durance, France
}

\begin{abstract}
MicroMegas detectors are versatile gaseous detectors which are used for ionizing particle detection. A MicroMegas detector consists of two adjacent gas-filled volumes. One volume acts as a drift region with an electric field operating in the ionization chamber regime, the second volume is the amplification region acting as a parallel-plate avalanche counter. The use of the microbulk technique allows the production of thin, radiation resistant, and low-mass detector with a highly variable gain. Such MicroMegas detectors have been developed and used in combination with neutron time-of-flight measurements for in-beam neutron-flux monitoring, fission and light-charged particle reaction cross section measurements, and for neutron-beam imaging. An overview of MicroMegas detectors for neutron detection and neutron reaction cross section measurements and related results and developments will be presented.
\end{abstract}

\section{Introduction}

Since the development of the first prototype, MicroMegas detectors [1] are used in many experiments in nuclear and particle physics. The MicroMegas was originally developed to cope with issues observed when existing gaseous detector families were used under high count rate. At present MicroMegas detectors are widely used in a variety of shapes [2]. These detectors usually have good position properties thanks to the often applied granular readout, have an excellent radiation hardness, and can cover large or even curved surfaces. With dedicated electronics, good timing properties can be achieved. Some examples of their use in high-energy experiments are CLAS12, ALTASNSW, and T2K. Other experiments concern X-ray detection from solar axions with the CAST project $[3,4]$, and even for muon tomography [5] as used in a radiography of a pyramid [6]. In nuclear physics a MicroMegas-based time-projection-chamber (TPC) for fission fragment tracking (NIFFTE) has been recently put in operation [7, 8].

The applicability of MicroMegas detectors for a diverse range of ionizing particles is due to the high range in possible gain, allowing to detect particles with very different energy deposits in the drift region. Therefore the versatility of the detector finds applications in detecting low-energy X-rays and even ultra-violet light in the $\mathrm{eV}$ range, to the detection of $100 \mathrm{MeV}$ fission fragments.

While MicroMegas detectors detect ionizing particles, an interesting application is its use as a neutron detector. The key for neutron detection is its conversion into a charged particle, either a light charged particle like a proton, an $\alpha$-particle, or a fission fragment. The application of MicroMegas detectors with neutron beams is not only

*e-mail: frank.gunsing@cea.fr useful at continuous neutron sources, typically as neutron monitors, but can be further extended to the use at pulsed time-of-flight facilities, such as GELINA at JRC-Geel [9], NFS at GANIL, or n_TOF at CERN [10]. For the neutron time-of-flight technique, the timing of the signal is important since it is a direct measure of the incident neutron energy.

MicroMegas stands for micro-mesh gaseous structure and is a parallel plate gaseous detector, composed of two gas volumes separated by a micromesh, which is a conductive electrode with many holes, such that the two gas volumes can be considered one for the gas flow. The first gas region is the drift volume. The ionizing particle crossing the drift zone ionizes the gas and releases primary electrons. It has an electric field which is in the ionization chamber regime, typically $1 \mathrm{kV} / \mathrm{cm}$ depending on the gas and the pressure. The field prevents the ions to recombine and the electrons drift to the micromesh, where they enter into the second gas volume. This amplification region has a much stronger electric field which is in the avalanche or proportional counter regime, typically $100 \mathrm{kV} / \mathrm{cm}$. Here the electrons are multiplied as secondary electrons and this part acts as a parallel-plate avalanche counter. This electronic avalanche considerably amplifies the electrical signal due to the initial energy deposition of the particle. The distance of the amplification region is much smaller than the drift region, typically $120 \mu \mathrm{m}$ for bulk, and $50 \mu \mathrm{m}$ for microbulk detectors. The electrons are collected at the anode, which often takes the role as readout device and is in many applications implemented as readout pixels, or orthogonal micro-strips in order to reduce the count rate per readout channel, and obtain position information. If position information is not needed, for example when used as 


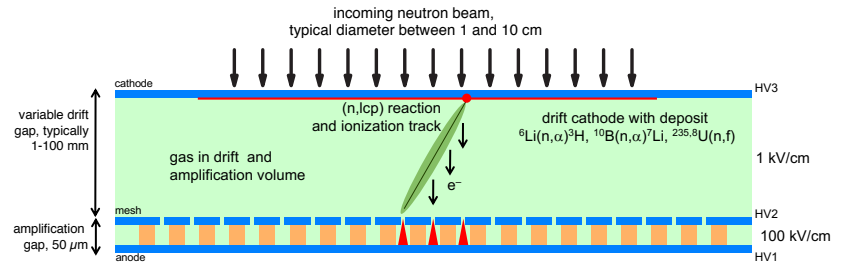

Figure 1. The principle of the microbulk MicroMegas detector as a neutron detector. The neutron beam has typically a diameter of several $\mathrm{cm}$, the detector covers the full neutron beam. The drift electrode is coated with material creating neutron-induced charged particles which ionize the gas in the drift region. The electrons are multiplied in the amplification region consisting of a chemically etched copper-coated kapton foil. The fast signal can be read out at either the mesh or the anode.

a simple counter, the anode can also be implemented as a single pad.

Earlier generation MicroMegas detectors are assembled mechanically. Especially the mesh-anode mounting is a critical step because of the strong forces due to the high electric field which may result in a varying meshanode distance and therefore an inhomogeneous electric field. Such problems are nowadays largely overcome by the use of the bulk technology [11]. The mesh structure and the anode readout strips or pixels are directly integrated in printed circuit board layers. This has resulted in much more robust detector elements. The bulk technique is at present the most widely spread production technique.

An even more recent development is the microbulk technology [12]. The starting point for a microbulk detector is a copper-coated kapton foil. Using optical masks and chemical etching, a mesh structure is created on one side of the foil. Small holes in the copper layer of typically 30 to $40 \mu \mathrm{m}$ diameter are created and the underlying kapton is removed. The opposite copper layer, the anode, remains usually as a single surface. The remaining kapton act as spacers between the two copper layers and the gasfilled space between the copper plates is the amplification region. The production technique of microbulk was inspired by that of GEM detectors [13], which together with MicroMegas form nowadays the principal components of the micropattern gas detector family (MPGD).

In figure 1 the principle of the MicroMegas detector is shown, here for a microbulk detector. Usual thicknesses are $50 \mu \mathrm{m}$ of kapton and $5 \mu \mathrm{m}$ copper on each side. Smaller gaps have been tested as well but appeared to produce less efficient avalanches at atmospheric pressure. Microbulk detectors show a very stable gain and excellent energy resolution [14]. The gas is continuously renewed in order to keep a constant ionizability. Figure 2 shows a microscope picture with the hole structure of a microbulk. Very recently, the production process of microbulk has been significantly improved with the use of laser direct imaging (LDI) instead of photolithography. LDI allows for the production of larger size detectors and a reduced interstrip region.

The in-beam material balance of such a detector is very low, most neutrons pass through the detector without inter-

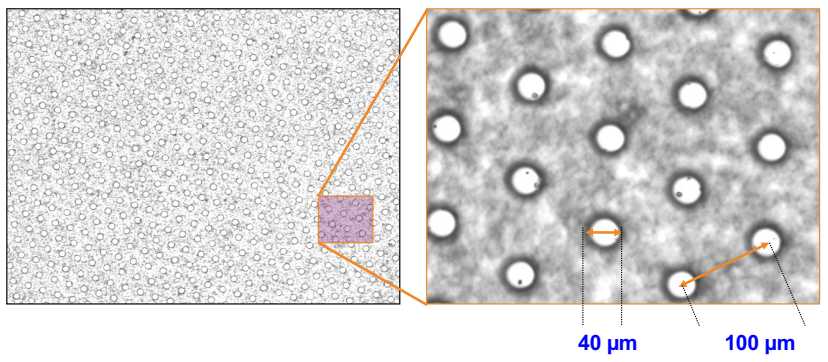

Figure 2. A microscope picture of the mesh of a microbulk detector. The patterns of the holes are clearly visible. The pitch value of $100 \mu \mathrm{m}$ and the hole diameter of $40 \mu \mathrm{m}$ are typical for microbulk detectors but may vary between generations and also depend on the kapton thickness and segmentation structure.

action. For this reason, such neutron detectors are sometimes called "transparent". Usually the strongest interaction comes from the neutron-to-charged particle converter. For neutron monitoring this is usually ${ }^{10} \mathrm{~B},{ }^{6} \mathrm{Li},{ }^{235} \mathrm{U}$, or ${ }^{238} \mathrm{U}$. These nuclei have neutron-induced reaction cross sections considered standard so that the number of incident neutrons can be accurately deduced. Also the transmission of neutrons through this material can be correctly determined and taken into account in the calculation of the ratio of the transmitted neutron flux with respect to the incident neutron flux. The three voltages are chosen such that the electric fields form the ionization chamber and proportional counter regimes in the drift and amplification region respectively.

\section{Use of MicroMegas with pulsed neutron beams}

Unlike charged particle beams which can be focused with magnets, neutron beams can only be shaped through collimation and therefore are used with diameters in the order of typically several centimeters. One of the challenges of accurate measurements of neutron-induced reactions, in particular for neutron capture measurements, is to determine the number of incident neutrons, (fluence or flux measurements), and their spatial distribution (beam profile) as a function of neutron energy deduced via measured time-of-flight. The MicroMegas detectors used with neutron beams can be distinguished in two types: the "single pad" detectors with a single read-out channel, used for neutron-induced reaction measurements, and the segmented detectors with multiple read-out channels, typically used for neutron imaging, but with the potential to be adapted for cross section measurements, including angular distributions.

\section{1 neutron reaction detectors}

The energy-density of the neutron as a function of neutron energy, shortly called the neutron flux, is an essential quantity to any cross section measurement. Without the need for the absolute number of neutrons, the relative 
change of the flux with neutron energy is usually the information needed to determined reaction cross sections from time-of-flight measurements. Thin in-beam neutron monitors are therefore the most appropriate detectors for this purpose. At the n_TOF facility the use of off-beam silicon detectors with an in-beam ${ }^{6} \mathrm{Li}$ foil has been successfully used since the start of the facility. Transparent microbulk MicroMegas neutron detectors have been developed for flux measurements at the $\mathrm{n}_{-} \mathrm{TOF}$ facility alongside other neutron flux detectors [15]. Nevertheless, MicroMegas detectors at $\mathrm{n} \_\mathrm{TOF}$ are usually employed for different purposes. Three formats of MicroMegas detectors have been developed. One with an active diameter of $35 \mathrm{~mm}$, one with a diameter of $60 \mathrm{~mm}$, used for setups with neutron beams from a small collimator, like for capture measurements, and one with an active diameter of $95 \mathrm{~mm}$, used for setups with a large collimator, typically for fission experiments [16], but also for $(\mathrm{n}, \alpha)$ measurements [17]. Pictures of these three formats are shown in figure 3. The transparency allows to stack several detectors in a single reaction chamber, in this way significantly decreasing the necessary beam time. For fission measurements, a reference sample can be part of the stack of detectors, resulting in very accurate fission ratios. An example of a stack of MicroMegas detectors is shown in figure 4. A variation of this single pad detector has been tested as well, by dividing the anode in four quarters of a circle, with the goal to achieve a quick way to find the beam center. Nevertheless, since the mesh covering the four quarter anodes is a single circle, an event in one quarter induces signals in the other quarters, making the data analysis cumbersome.

\section{2 neutron beam-profile detectors}

The neutron beam profile detectors were until recently implemented as bulk detectors on PCB support. Neutron capture measurements usually consist of a capture sample in a pulsed neutron beam, viewed by gamma-ray detectors outside the beam. This results in a capture gamma-ray spectra as a function of neutron time-of-flight. Such a measurement is usually normalized by a reference sample placed in the same position as the capture sample. The normalization is extracted from the reference sample in a very limited energy range (usually a "saturated" resonance) and then applied to the capture sample over the entire energy range. Therefore it is important to know if the beam pro-

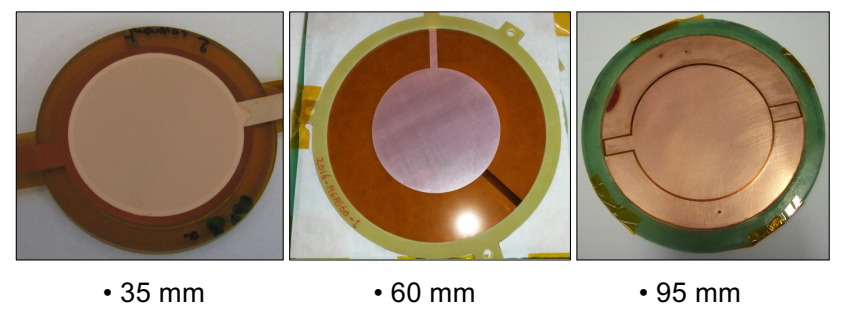

Figure 3. Three diameters of 35, 60 and $95 \mathrm{~mm}$ for single pad MicroMegas detectors, as used in n_TOF experiments. file, or more precisely the fraction of neutrons incident on the sample, changes with neutron energy.

In order to measure the energy-dependence of the neutron beam profile, a first MicroMegas detector in the form of a bulk detector with mono-directional anode read-out strips, developed for the CAST experiment [4], has been used at $n \_$TOF [18]. The detector consisted of a segmented anode of 50 strips of $1.5 \mathrm{~mm}$ wide and used a $595 \mathrm{~nm}{ }^{6} \mathrm{LiF}$ foil as a neutron converter. By rotating the detector around the axis of the neutron beam, data were taken for $0^{\circ}, 30^{\circ}$ and $90^{\circ}$. From a combined analysis of the data at different angles the neutron beam profile could be extracted [18]. Within the measurement uncertainties the profile was found to be in agreement with FLUKA simulations in the used energy ranges.

A second beam profile detector, based on a prototype for CAST, had orthogonal X- and Y- strips, 106 in each direction, and was used in 2009 at n_TOF. The energydependent profile was determined from coincidences in the $\mathrm{X}$ and $\mathrm{Y}$ strips, together with the time-of-flight information. The drift electrode was covered with a ${ }^{10} \mathrm{~B}_{4} \mathrm{C}$ layer to detect the neutron-induced light charged particles from the ${ }^{10} \mathrm{~B}(\mathrm{n}, \alpha)$ reaction. The data acquisition was based on the Gassiplex multiplexer. The timing information of this device did not allow for track reconstruction to find the neutron interaction point. Extensive tests and simulations were performed for this detector. A typical beam image for an energy interval at low neutron energy, taken from ref. [19], is shown in the left panel of figure 5 .

The use of such an image is in the first place to identify the absolute position of the neutron beam spot, which depends on the configuration and placement of the collimators. Then for capture measurements, the part of beam that impinges on the capture sample can be determined. This is usually referred to as the beam interception factor. For

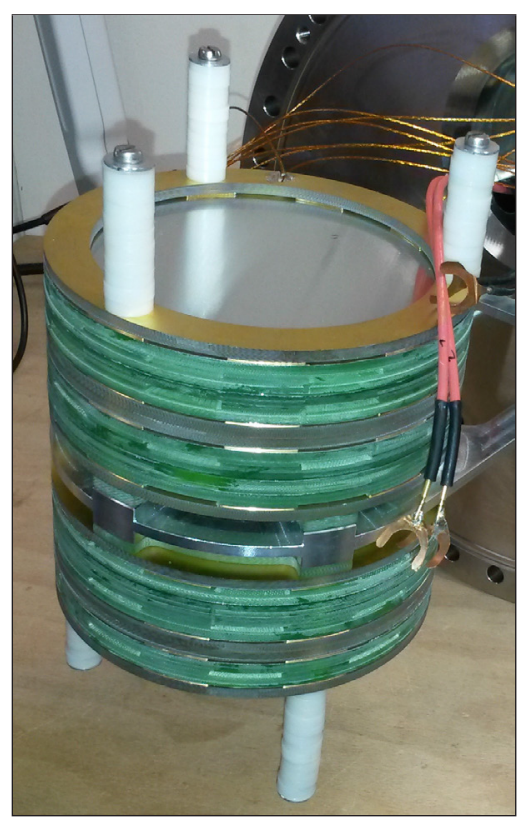

Figure 4. An example of a stack of MicroMegas detectors on a mounting structure for insertion in a reaction chamber. 

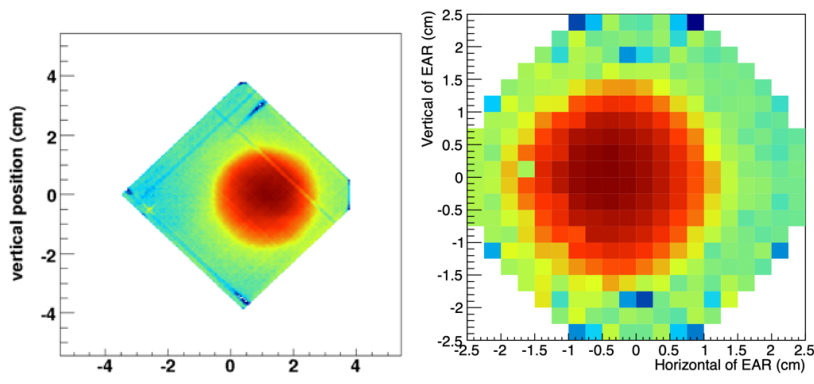

Figure 5. Neutron beam images obtained using a MicroMegas detector with XY strips (left panel), and using a pixelized MicroMegas (right panel). Figures are from ref. [19].

this it is possible to find an analytical function describing the image, but it is by easier to integrate the counts in the two-dimensional histogram over the shape of the capture sample. After this XY MicroMegas detector a specially designed pixelized detector was used. The detector was realized in a PCB, and consisted of 308 pixels, disposed with a pitch of $2.5 \mathrm{~mm}$ and enveloping a circular shape of $5 \mathrm{~cm}$ diameter. The resulting image, shown in the right panel of figure 5 , has evidently a much worse resolution because of the larger pixel sizes.

A breakthrough for the strip detectors came with the advent of the possibility to segmentize not only the anode, but also the mesh of microbulk detector. This was the result of a prototype evolution made within an RD51 project. In this way, a real 2D structure was achieved without addition of extra layers, resulting in a very low-mass neutron profiler, practically transparent to neutrons. The very first detector with a transparent stripped microbulk detector was developed with $60+60$ strips of $1 \mathrm{~mm}$ wide, and was used for measurements at GELINA and then at n_TOF. One side with strips is shown in figure 6 . The detector was coupled to a data acquisition system based on the AGET ASIC chip [21]. This system offers the possibility of an auto-trigger mode and records the time difference between the strips recording a signal, allowing for the reconstruction of the track of the charged particles in the detector gas and therefore the interaction point of the incident neutrons. A full description of this detector and results can be found in ref. [20].

\section{Outlook}

The new technical possibilities of microbulk MicroMegas detectors with both a segmented anode and mesh, combined with high precision LDI patterns, allow in principle for the unification of the transparent single-pad detectors, used for beam monitoring, fission, and $(\mathrm{n}, \alpha)$ or other light charged particle measurements, and the XY-type detectors, used for beam imaging. In addition, the distinct observation of the arrival times induced by a single track, as already observed in ref. [18] and further exploited in

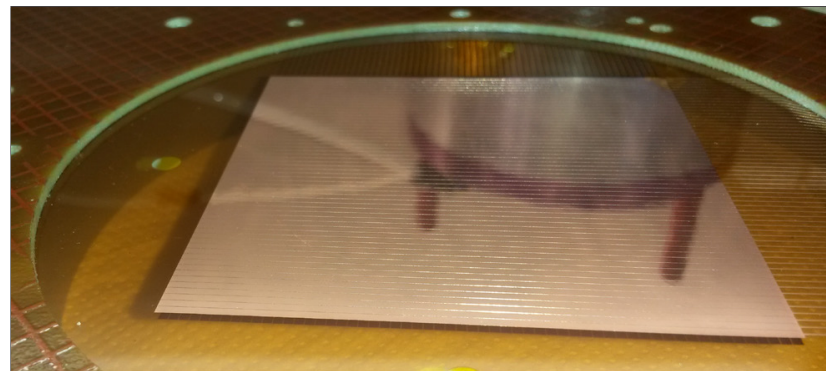

Figure 6. One side of a double sided segmented microbulk, showing the $1 \mathrm{~mm}$ wide strips, used for precise neutron beam imaging as function of neutron energy [20].

ref. [20], may open the possibility of a more precise track reconstruction, in the sense of a simplified time projection chamber (TPC). This may lead to the use of a MicroMegas detector for the measurement of angular distributions of neutron-induced charged particle reactions.

\section{References}

[1] Y. Giomataris et al., Nucl. Instr. Methods A 376, 29 (1996)

[2] (in French), Scintillations 97-98 (2019), http://irfu.cea.fr/scintillations

[3] P. Abbon et al., New Journal of Physics 9, 170 (2007)

[4] The CAST Coll., Nature Physics 13, 584 (2017)

[5] S. Bouteille et al., Nucl. Instr. Methods A 834, 223 (2016)

[6] K. Morishima et al., Nature 552, 386 (2017)

[7] R.J. Casperson et al., Phys. Rev. C 97, 034618 (2018)

[8] (2019), see contributions from: N. Walsh, N. Bowden, these proceedings

[9] J. Heyse et al. (2019), these proceedings

[10] E. Chiaveri et al. (2019), these proceedings

[11] I. Giomataris et al., Nucl. Instr. Methods A 560, 405 (2006)

[12] S. Andriamonje et al., J. Instr. 5, P02001 (2010)

[13] F. Sauli, Nucl. Instr. Methods A 386, 531 (1997)

[14] F. Iguaz et al., Physics Procedia 37, 448 (2012)

[15] M. Sabaté-Gilarte et al., Eur. Phys. J. A 53, 210 (2017)

[16] (2019), see contributions from: S. Chasapoglou, Z. Eleme, V. Michalopoulou, A. Stamatopoulos, A. Tsinganis, these proceedings

[17] J. Praena et al., Phys. Rev. C 97, 064603 (2018)

[18] J. Pancin et al., Nucl. Instr. Methods A 524, 102 (2004)

[19] F. Belloni, Tech. rep. (2014), IRFU-14-07

[20] M. Diakaki et al., Nucl. Instr. Methods A 903, 46 (2018)

[21] E. Pollacco et al., Nucl. Instr. Methods A 887, 81 (2018) 